\title{
Taxa de Desenvolvimento de Força em Diferentes Velocidades de Contrações Musculares
}

\author{
Rate of Force Development in Different Muscle \\ Contraction Velocities
}

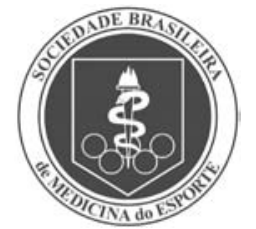

Artigo Original

Rogério Bulhões Corvino

Fabrizio Caputo

Anderson Castelo de Oliveira

Camila Coelho Greco

Benedito Sérgio Denadai

Laboratório de Avaliação da Performance Humana, Unesp Rio Claro, SP.

\section{Endereço para correspondência: Benedito Sérgio Denadai \\ Laboratório de Avaliação da \\ Performance Humana - Instituto \\ de Biociências - Av. 24A, 1.515, Bela \\ Vista - 13506-900 - \\ Rio Claro, SP - Brasil \\ E-mail: bdenadai@rc.unesp.br}

Submetido em: 11/11/2008

Versão final recebida em: 27/04/2009

Aceito em: 13/05/2009

\begin{abstract}
RESUMO
O objetivo deste estudo foi comparar a taxa de desenvolvimento de força (TDF) nas contrações isométrica e isocinética concêntrica a $60^{\circ} \cdot \mathrm{s}^{-1}$ e $180^{\circ} \cdot \mathrm{s}^{-1}$. Quatorze indivíduos do gênero masculino (idade $=23,1 \pm 2,8$ anos; estatura $=174 \pm 31,3 \mathrm{~cm}$; massa corporal $=81 \pm 12 \mathrm{~kg}$ ) realizaram inicialmente uma familiarização ao equipamento isocinético. Posteriormente, os indivíduos realizaram em ordem randômica cinco contrações isocinéticas máximas para os extensores do joelho a $60^{\circ} \mathrm{s}^{-1} \mathrm{e} 180^{\circ} \mathrm{s}^{-1}$ para determinar o torque máximo concêntrico (TMC) e duas contrações isométricas máximas de $3 \mathrm{~s}$ para determinar o torque máximo isométrico (TMI). O TMI (301,4 $\pm 56,0 \mathrm{~N}$.m) foi maior do que o TMC a $60^{\circ} \cdot \mathrm{s}^{-1}(239,8 \pm 42,2 \mathrm{~N} \cdot \mathrm{m})$ e $180^{\circ} . \mathrm{s}^{-1}(175,0 \pm 32,5 \mathrm{~N} . \mathrm{m})$. OTMC a $60^{\circ} \cdot \mathrm{s}^{-1}$ foi maior do que o TMC a $180^{\circ} \mathrm{s}^{-1}$. Para os intervalos de $0-30 \mathrm{~ms}$ e $0-50 \mathrm{~ms}$, a TDF na condição isométrica $\left(1.196,6 \pm 464,6\right.$ e 1.326,5 $\pm 514,2 \mathrm{~N} . \mathrm{m} . \mathrm{s}^{-1}$, respectivamente) foi similar à TDF a $60^{\circ} \mathrm{s}^{-1}(1.035,4 \pm$ 446,2 e $\left.1.134,3 \pm 448,4 \mathrm{~N} \cdot \mathrm{m} \cdot \mathrm{s}^{-1}\right)$ e maior do que a $180^{\circ} \cdot \mathrm{s}^{-1}\left(656,7 \pm 246,6\right.$ e 475,2 $\left.\pm 197,9 \mathrm{~N} \cdot \mathrm{m} . \mathrm{s}^{-1}\right)$, sendo ainda que a TDF na contração concêntrica a $180^{\circ} . \mathrm{s}^{-1}$ foi menor do que a $60^{\circ} \mathrm{s}^{-1}$. No intervalo de 0-100ms, a TDF da contração isométrica $\left(1.248,8 \pm 417,4 \mathrm{~N} \cdot \mathrm{m}^{-1} \mathrm{~s}^{-1}\right)$ foi maior que a obtida na contração isocinética rápida $(909,2$ $\left.\pm 283,4 \mathrm{~N} \cdot \mathrm{m} \cdot \mathrm{s}^{-1}\right)$. A TDF obtida na contração isocinética lenta $\left(1.005,4 \pm 247,7 \mathrm{~N} \cdot \mathrm{m} . \mathrm{s}^{-1}\right)$ foi similar à obtida na contração isométrica e na concêntrica isocinética rápida. No intervalo 0-150ms, a TDF isométrica (1.084,2 \pm $\left.332,1 \mathrm{~N} . \mathrm{m} . \mathrm{s}^{-1}\right)$ foi maior do que as concêntricas $\left(60^{\circ} \mathrm{s}^{-1}\right.$ e $\left.180^{\circ} . \mathrm{s}^{-1}\right)\left(834,8 \pm 184,2\right.$ e $767,6 \pm 201,8 \mathrm{~N}$. m. $\mathrm{s}^{-1}$, respectivamente), não existindo diferenças entre estas duas últimas. Conclui-se que a TDF é dependente do tipo e da velocidade de contração, suportando a hipótese de que maiores velocidades de contração acarretam maior inibição do drive neural no início do movimento.
\end{abstract}

Palavras-chave: contração concêntrica, velocidade angular, isocinética.

\begin{abstract}
The objective of this study was to compare the maximal rate of force development (RFD) at maximal isometric and isokinetic concentric contractions at $60^{\circ} . \mathrm{s}^{-1}$ and $180^{\circ} . \mathrm{s}^{-1}$. Fourteen active males (age $=23.1 \pm$ 2.8 years; height $=174 \pm 31.3 \mathrm{~cm}$ and body mass $=81 \pm 12 \mathrm{~kg}$ ) volunteered to participate in this study. During the first visit, subjects performed a familiarization to the isokinetic equipment. During the second visit, subjects performed in random order 5 maximal isokinetic concentric contractions for knee extensors at each angular velocity $\left(60\right.$ and $180^{\circ} . s^{-1}$ ) to determine maximal concentric torque (MCT) and 2 maximal isometric contractions of $3 \mathrm{~s}$ to determine maximal isometric torque (MIT). The MIT (301.4 $\pm 56.0 \mathrm{~N} . \mathrm{m})$ was higher than MCT at $60^{\circ} . \mathrm{s}^{-1}(239.8 \pm 42.2 \mathrm{~N} . \mathrm{m})$ and $180^{\circ} . \mathrm{s}^{-1}(175.0 \pm 32.5 \mathrm{~N} . \mathrm{m})$. The MCT at $60^{\circ} \mathrm{s}^{-1}$ was higher than MCT at $180^{\circ} . \mathrm{s}^{-1}$. At intervals of $0-30 \mathrm{~ms}$ and $0-50 \mathrm{~ms}$, the RFD at isometric contraction $(1196.6 \pm 464.6$ and $1326.5 \pm$ $514.2 \mathrm{~N} . \mathrm{m} . \mathrm{s}^{-1}$, respectively) was similar to that obtained during concentric contraction at $60^{\circ} \mathrm{s}^{-1}(1035.4 \pm 446.2$ N.m. $s^{-1}$ and $1134.3 \pm 448.4 \mathrm{~N} . \mathrm{m} . \mathrm{s}^{-1}$, respectively) and higher than that obtained during concentric contraction at $180^{\circ} . \mathrm{s}^{-1}\left(656.7 \pm 246.6 \mathrm{~N} \cdot \mathrm{m} . \mathrm{s}^{-1}\right.$ and $475,2 \pm 197,9 \mathrm{~N} \cdot \mathrm{m} . \mathrm{s}^{-1}$, respectively). For the interval of 0-100ms, RFD at isometric contraction $\left(1248.7 \pm 417.4 \mathrm{~N} . \mathrm{m}^{-1} \mathrm{~s}^{-1}\right)$ was higher than that obtained during concentric contractions at $180^{\circ} . \mathrm{s}^{-1}\left(909.2 \pm 283.4 \mathrm{~N} \cdot \mathrm{m} . \mathrm{s}^{-1}\right)$, and similar to concentric contractions at $60^{\circ} . \mathrm{s}^{-1}\left(1005.4 \pm 247.7 \mathrm{~N} \cdot \mathrm{m} . \mathrm{s}^{-1}\right)$. However, for the interval of 0-150ms, RFD at isometric contraction (1084.2 $\left.\pm 332.1 \mathrm{~N} . \mathrm{m}^{-1} \mathrm{~s}^{-1}\right)$ was higher than at $60^{\circ} . \mathrm{s}^{-1}\left(834.8 \pm 184.2 \mathrm{~N} \cdot \mathrm{m} . \mathrm{s}^{-1}\right)$ and $180^{\circ} . \mathrm{s}^{-1}\left(767.6 \pm 767.6 \mathrm{~N} \cdot \mathrm{m} . \mathrm{s}^{-1}\right)$. It can be concluded that RFD is dependant on the contraction type and velocity, supporting the hypothesis that higher speed of contraction generates higher neural drive inhibition at the beginning of the movement.
\end{abstract}

Keywords: concentric contraction, angular speed, isokinetics. 


\section{INTRODUÇÃO}

No âmbito esportivo, existem muitas modalidades ou, mais especificamente, componentes técnicos que não utilizam em seus movimentos todo o potencial de força (i.é., pico de força) existente na musculatura recrutada (p.ex., chute no caratê, saída do bloco nos 50m na natação, soco no boxe, entre outros). Isso ocorre em função de essas atividades serem compostas de movimentos muito rápidos (até $200 \mathrm{~ms}$ ), ou seja, movimentos explosivos, não existindo o tempo necessário para se obter o pico de força existente na musculatura recrutada, que é atingido por volta de 200-300 ms $s^{(1)}$.

A força explosiva muscular é bastante dependente da taxa de aumento da força em um dado intervalo de tempo no início da contração muscular, sendo os valores máximos dessa taxa alcançados em um período de tempo entre 100 e 300ms ${ }^{(2)}$. Essa taxa é conhecida como taxa de desenvolvimento de força (TDF) e é obtida através da razão entre a variação da força e a variação do tempo. Assim, em atletas que utilizam em suas modalidades contrações musculares explosivas, a TDF tem sido considerada um importante parâmetro para mensurar o desempenho neuromuscular.

A TDF é influenciada por diferentes fatores, como as propriedades musculares (tamanho muscular, área relativa das fibras rápidas e a composição da isoforma da miosina de cadeia pesada) ${ }^{(3,4)}$, distribuição das fibras musculares ${ }^{(4-6)}$ e fatores neurais, como a magnitude de produção eferente do motoneurônio na fase inicial de contração ${ }^{(7-9)}$, a frequência de disparos e o recrutamento dos motoneurônios ${ }^{(2,7,9-11)}$. Além desses fatores, o nível de complacência das estruturas relacionadas com a transmissão da força também é importante para explicar as diferenças na TDF ${ }^{(12)}$. Em movimentos como o salto vertical, a contribuição desse fator pode chegar a $30 \%{ }^{(13)}$.

Nesse sentido, é importante destacar que poucos estudos analisaram o comportamento da TDF em contrações musculares realizadas em diferentes velocidades ${ }^{(14)}$, sendo esta mais frequentemente medida em condições isométricas( ${ }^{(2)}$. Apesar disso, a TDF tem sido considerada um importante parâmetro para quantificar a habilidade do sistema neuromuscular de exercer ações musculares explosivas realizadas com contrações dinâmicas $(2,3,5,15,16)$. Como não se conhecem os efeitos do tipo e velocidade de contração muscular sobre a TDF, e particularmente a influência de cada um dos fatores neuromusculares sobre a TDF nessas diferentes condições, a utilização dessa variável na avaliação de atletas deveria ser realizada com cautela.

Dados na literatura sugerem que maiores velocidades de contração acarretam inibição do drive neural e, possivelmente, a quantidade de torque gerado nos primeiros momentos da contração pode ser diminuída sob condições dinâmicas, particularmente as realizadas em alta velocidade ${ }^{(1)}$. Com base nesses dados, é possível hipotetizar que a TDF será maior na condição isométrica, quando comparada com a condição dinâmica, como, também, que a TDF em contração de baixa velocidade $\left(60^{\circ} . \mathrm{s}^{-1}\right)$ será maior que em velocidade mais alta $\left(180^{\circ} . \mathrm{s}^{-1}\right)$, uma vez que existem alterações neurais entre tipos de contração e entre velocidades de contração(1) . Com isso, o objetivo deste estudo foi comparar a TDF nas contrações isométricas e isocinéticas concêntricas a $60^{\circ} . \mathrm{s}^{-1}$ e $180^{\circ} . \mathrm{s}^{-1}$.

\section{MÉTODOS}

\section{Sujeitos}

Participaram deste estudo 14 voluntários do sexo masculino, aparentemente saudáveis, sem prática regular de atividades físicas, com as seguintes características: idade $=23,1 \pm 2,8$ anos; estatura $=174 \pm 31,3 \mathrm{~cm}$ e; massa corporal $=81 \pm 12 \mathrm{~kg}$. Após ser informados textual e verbalmente sobre os objetivos e a metodologia deste estudo, assinaram um termo de consentimento livre e esclarecido. O protocolo deste estudo foi aprovado pelo Comitê de Ética em Pesquisa do IB - Unesp - Rio Claro (144/2008).

\section{Delineamento experimental}

Os indivíduos compareceram ao laboratório em duas diferentes ocasiões. Na primeira visita os indivíduos realizaram uma familiarização ao teste e foram realizadas as medidas antropométricas. Na segunda visita os voluntários realizaram os testes máximos no dinamômetro isocinético para a determinação do torque máximo isométrico (TMI) e concêntrico (TMC) nas velocidades de 60 e 180 ${ }^{\circ} \mathrm{s}^{-1}$ e da TDF nas condições isométricas e concêntricas nas velocidades em 60 e $180^{\circ} . \mathrm{s}^{-1}$. Os testes para determinação do TMC e da TDF nas contrações isométricas e isocinéticas foram realizados em ordem aleatória. O dinamômetro isocinético foi calibrado antes de cada sessão de teste de acordo com os procedimentos prescritos pelo fabricante.

\section{Medidas antropométricas}

As medidas antropométricas foram realizadas com os voluntários descalços vestindo apenas roupas leves (bermuda e camiseta). Para medir a massa corporal foi utilizada uma balança (Filizola) com resolução de $100 \mathrm{~g}$. A estatura foi medida por um estadiômetro com precisão de 0,5cm localizado na própria balança.

\section{Contrações isométricas}

Foram feitas duas contrações isométricas máximas de extensão do joelho em um dinamômetro isocinético (Biodex System 3, Biodex Medical Systems, Shirley, NY, EUA). A articulação do joelho foi fixada a $75^{\circ}$ para a realização da contração isométrica por um período de $3 \mathrm{~s}$, com o sujeito sentado e seu quadril fixado numa angulação de $90^{\circ}$. Houve um período de três minutos de recuperação entre as contrações isométricas. Foi considerado o maior valor como sendo o TMI.

\section{Contrações isocinéticas concêntricas}

Para a determinação do TMC e da TDF foram realizadas cinco contrações máximas concêntricas dos extensores do joelho no dinamômetro isocinético nas duas velocidades analisadas (60 e $\left.180^{\circ} \cdot \mathrm{s}^{-1}\right)$. A amplitude de movimento foi de $10^{\circ}$ a $90^{\circ}$, considerando que $0^{\circ}$ corresponde à extensão completa do joelho. Ocorreu um período de três minutos de recuperação entre as contrações realizadas nas diferentes velocidades. Foi utilizado somente o membro dominante para a mensuração do torque e a ordem de realização das contrações nas diferentes velocidades foi randômica. O TMC correspondeu ao maior valor de torque produzido a partir dos cinco esforços.

\section{Determinação da taxa de desenvolvimento de força}

Os dados de torque das contrações isométricas e isocinéticas foram coletados a partir do software do dinamômetro isocinético e analisados no software MatLab 6.5. Os dados de torque de todas as contrações válidas foram filtrados (filtro Butterworth, quarta ordem), e a partir do início da contração (estipulado como sendo o valor de torque acima de $\left.8 \mathrm{~N} . \mathrm{m}^{(2)}\right)$ foram calculados os valores de taxa de desenvolvimento de força (TDF) (N.m.s $\mathrm{s}^{-1}$ ) nos intervalos de $1 \mathrm{~ms}$ até $30 \mathrm{~ms}, 50 \mathrm{~ms}$, $100 \mathrm{~ms}$ e $150 \mathrm{~ms}$. A TDF foi considerada como a inclinação média da curva momento-tempo nos intervalos de tempo de 0-30, 0-50, 0-100 e 0-150 ms relativos ao início da contração(2).

\section{ANÁLISE ESTATÍSTICA}

Os dados estão expressos como média \pm DP. Após verificação da normalidade dos dados (teste de Shapiro-Wilk) foi utilizada a ANOVA One-way, complementada pelo teste de Tukey. As correlações foram determinadas através do teste de correlação de Pearson. 0 nível de significância foi estabelecido em $p \leq 0,05$. 


\section{RESULTADOS}

A figura 1 apresenta os valores médios \pm DP do TMI e do TMC obtido em 60 e $180^{\circ} . \mathrm{s}^{-1}$. O TMl foi significantemente maior do que o TMC em 60 e $180^{\circ} . s^{-1}(p<0,05)$. OTMC em 60 $0^{\circ} s^{-1}$ foi significantemente maior do que o TMC em $180^{\circ} . s^{-1}(p<0,05)$.

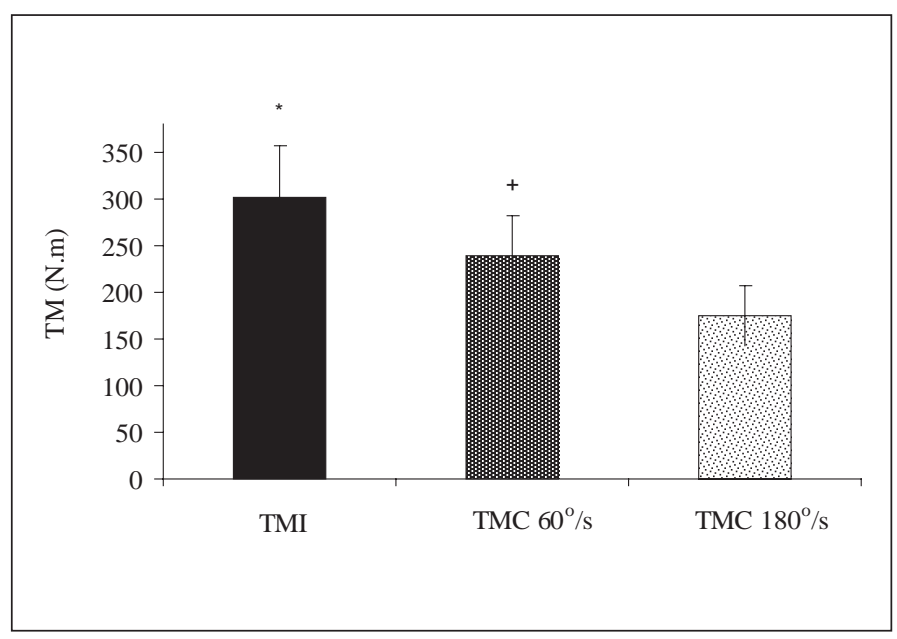

Figura 1. Valores médios \pm DP do torque máximo isométrico (TMI) e do torque máximo concêntrico (TMC) obtido em 60 e $180^{\circ} . \mathrm{s}^{-1}$. ${ }^{*} \mathrm{p}<0,05$ em relação às condições concêntrica a $60^{\circ} . \mathrm{s}^{-1}$ e concêntrica a $180^{\circ} . \mathrm{s}^{-1}$. $+\mathrm{p}<0,05$ em relação à condição concêntrica a $180^{\circ} \cdot \mathrm{s}^{-1}$. $\mathrm{N}=14$

A figura 2 apresenta os valores médios \pm DP da TDF obtidos nas condições isométrica, concêntrica a $60^{\circ} .5^{-1}$ e concêntrica a $180^{\circ} .5^{-1}$. Para os intervalos de 0-30ms e 0-50ms, a TDF na condição isométrica foi similar à TDF a $60^{\circ} . s^{-1}(p>0,05)$ e maior do que a $180^{\circ} \cdot s^{-1}(p<0,05)$, sendo ainda que a TDF na contração concêntrica rápida $\left(180^{\circ} . \mathrm{s}^{-1}\right)$ foi menor do que na lenta $\left(60^{\circ} . \mathrm{s}^{-1}\right)(\mathrm{p}<0,05)$. No intervalo $0-100 \mathrm{~ms}$, a TDF obtida na contração isométrica foi significantemente maior que a obtida na contração isocinética rápida $\left(180^{\circ} . s^{-1}\right)(p<0,05)$. A TDF na contração isocinética lenta $\left(60^{\circ} . \mathrm{s}^{-1}\right)$ foi similar à obtida na contração isométrica e na concêntrica isocinética rápida $\left(180^{\circ} \mathrm{s}^{-1}\right)(\mathrm{p}>0,05)$. No intervalo de $0-150 \mathrm{~ms}$, a TDF isométrica foi maior do que a das concêntricas $\left(60^{\circ} .5^{-1}\right.$ e $\left.180^{\circ} .^{-1}\right)$, não existindo diferenças entre estas duas últimas ( $p>0,05)$.

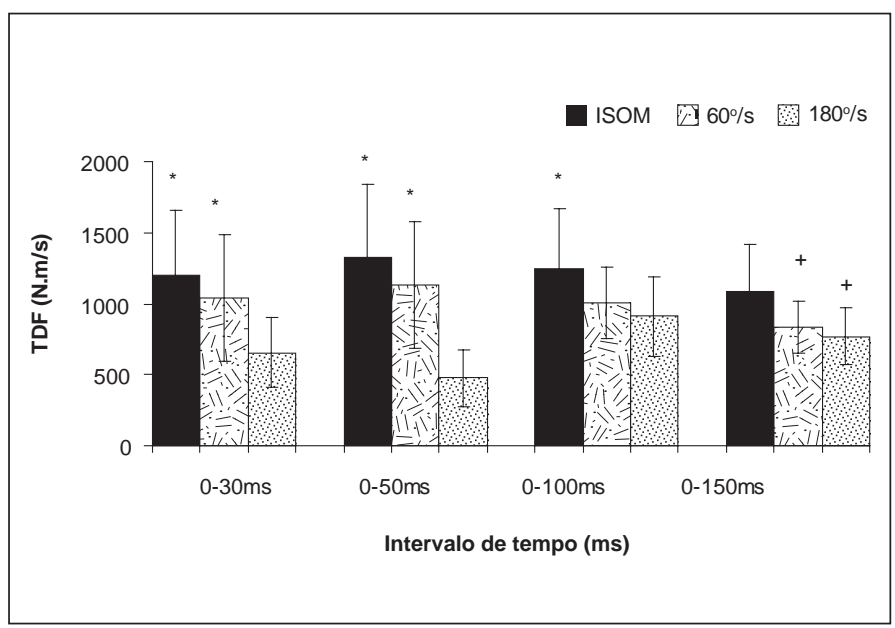

Figura 2. Valores médios \pm DP da taxa de desenvolvimento de força (TDF) obtida nas condições isométrica, concêntrica a $60^{\circ} \cdot \mathrm{s}^{-1}$ e concêntrica a $180^{\circ} \cdot \mathrm{s}^{-1}$. ${ }^{*} \mathrm{p}<0,05$ em relação à condição concêntrica a $180^{\circ} \cdot \mathrm{s}^{-1}$. $+\mathrm{p}<0,05$ em relação à condição isométrica. $\mathrm{N}=14$.
Houve correlação significante da TDF obtida na condição isométrica com a TDF obtida nas condições concêntricas a $60^{\circ} \cdot \mathrm{s}^{-1}(r=0,88)$ e $180^{\circ} \cdot s^{-1}(r=0,60)(p<0,05)$. Da mesma forma, houve correlação significante da TDF obtida nas condições concêntricas a $60^{\circ} . \mathrm{s}^{-1}$ e $180^{\circ} . \mathrm{s}^{-1}$ $(r=0,78)(p<0,05)$. Não houve correlação significante da TDF obtida na condição isométrica com o TMI $(r=0,31)$ e da TDF nas condições concêntricas a $60^{\circ} \cdot \mathrm{s}^{-1}$ e $180^{\circ} \cdot \mathrm{s}^{-1} \mathrm{com}$ o TMC obtido a $60^{\circ} \cdot \mathrm{s}^{-1}(\mathrm{r}=0,51) \mathrm{e}$ $180^{\circ} \cdot s^{-1}(r=0,48)(p>0,05)$.

\section{DISCUSSÃO}

O principal achado deste estudo foi que, de maneira geral, os valores de TDF são dependentes do tipo e da velocidade de contração utilizados. Esses dados confirmam as hipóteses iniciais do estudo, sugerindo que altas velocidades de contração inibem o drive neural, podendo também influenciar nos valores máximos de torque obtidos.

No presente estudo, os valores de TMI foram maiores do que o TMC $\left(60^{\circ} \mathrm{s}^{-1} \mathrm{e} 180^{\circ} \mathrm{s}^{-1}\right)$. Além disso, o maior TMC foi obtido na menor velocidade de contração $\left(60^{\circ} . \mathrm{s}^{-1}\right)$. Esses dados estão de acordo com os observados na literatura, que compararam o torque máximo em condições similares ${ }^{(11,17,18)}$, reforçando que a existência de movimento articular determina valores diferentes de torque, como também que, para contrações concêntricas, a relação entre torque máximo e velocidade de contração é inversa(19).

Com relação aos valores de TDF, em geral esta variável também foi maior nas condições de ausência de movimento articular ou de menor velocidade de contração. Esses dados diferem dos obtidos por Wilson et al. ${ }^{(14)}$, que analisaram o exercício de saltos em diferentes condições. Nesse estudo, os autores utilizaram um equipamento que permitia a mensuração da força utilizando-se contração isométrica e saltos com e sem contramovimento. Os autores verificaram que a força isométrica $(1.989 \mathrm{~N})$ foi maior do que a concêntrica (1.707N). No entanto, a TDF foi maior na contração concêntrica do que na isométrica, tanto a $110^{\circ}$ $\left(19.266 \mathrm{~N} . \mathrm{s}^{-1}\right.$ e $14.059 \mathrm{~N} . \mathrm{s}^{-1}$, respectivamente) quanto a $150^{\circ}\left(23.949 \mathrm{~N} . \mathrm{s}^{-1}\right.$ e 11.662 N. $\mathrm{s}^{-1}$, respectivamente). Assim, ao comparar as condições isométrica e concêntrica, os maiores valores de TDF foram produzidos na condição de menor produção de força.

Ao comparar os valores de força e de TDF com os do presente estudo, nota-se que os obtidos por Wilson et al.(14) são bem maiores. Isso em parte pode ser explicado pelas diferentes condições de realização das contrações. Enquanto que no trabalho de Wilson et al. ${ }^{(14)}$ as condições permitiam aceleração do movimento, no presente estudo a contração utilizada foi realizada em velocidade constante. Da mesma forma, a condição de realização da contração isométrica foi diferente também entre os estudos, em termos de angulação e posição corporal. Assim, a comparação direta entre os estudos deve ser feita com cautela. De qualquer forma, os maiores valores de TDF encontrados na condição concêntrica no estudo de Wilson et al.(14) podem estar relacionados com a possibilidade de aceleração do movimento. Além disso, a magnitude da diferença nos valores de torque do estudo de Wilson et al. ${ }^{(14)}(14 \%)$ é inferior à do presente trabalho (20-50\%).

Entre os principais fatores que influenciam a TDF estão os musculares, como o tamanho, a área e a distribuição das fibras musculares, como também os neurais, como os estímulos eferentes do motoneurônio na fase inicial de contração, a frequência de disparos e o recrutamento dos motoneurônios. Além da importância para a TDF, esses fatores também são importantes para a produção da força máxima muscular. No entanto, os valores máximos de TDF podem ser atingidos antes que os níveis máximos de força muscular sejam produzidos. Assim, um aumento na TDF pode ser bastante importante para maior nível de força produzido no início da contração(2). Entretanto, a ausência de correlação significante entre a TDF e o TMC em nosso estudo confirma que os fatores neuromusculares que determinam essas duas variáveis podem ser diferentes. 
Estudos sugerem que a quantidade máxima de torque produzida pode ser menor em maiores velocidades de contração em função da inibição do drive neural(1). Assim, como a TDF também é influenciada por mecanismos neurais relacionados com os estímulos elétricos, essa inibição pode auxiliar na explicação dos menores valores de TDF encontrados nas contrações dinâmicas e nas maiores velocidades. Interessantemente, a correlação entre a TDF isométrica e a concêntrica em baixa velocidade $\left(60^{\circ} . s^{-1} ; r=0,88\right)$ foi maior do que obtida entre a isométrica e a concêntrica em alta velocidade $\left(180^{\circ} . \mathrm{s}^{-1} ; \mathrm{r}=0,60\right) \mathrm{e}$ também entre as duas concêntricas $(r=0,78)$. Esse comportamento concorda com dados obtidos anteriormente, quando se analisou a relação entre o torque máximo isométrico e o isocinético medido em diferentes velocidades. Knapik e Ramos ${ }^{(20)}$ verificaram uma relação elevada entre o teste isométrico e testes realizados em baixas velocidades. No entanto, quanto maior a diferença entre as velocidades, menores foram os níveis de correlação encontrados. Os autores sugerem que, quanto maior a diferença entre as velocidades, mais diferentes serão os padrões de recrutamento neural e coordenação. Assim, esses dados, juntamente com os do presente estudo, sugerem que a velocidade de contração, além de influenciar nos valores de TDF, pode também modificar os fatores que a determinam.

Finalmente, as possíveis influências (isoladas ou associadas) do grupo muscular e do estado e/ou período de treinamento sobre a TDF medida em diferentes condições ainda não são conhecidas. Assim, os dados aqui obtidos devem ser analisados considerando-se os grupos musculares (extensores do joelho) e o estado de treinamento dos indivíduos analisados (não treinados). Apenas para exemplificar, pode-se apontar que os efeitos do treinamento de força sobre os componentes central (neural) e periférico (músculo) do sistema neuromuscular são dependentes, entre outros, da relação intensidade vs. volume, tipo de contração muscular (concêntrica, excêntrica e isométrica) e tempo de treinamento(21). Portanto, a influência do tipo e velocidade de contração muscular sobre a TDF pode depender das características do treinamento realizado no período imediatamente anterior ao da coleta de dados.

\section{CONCLUSÃO}

Podemos concluir que, em indivíduos não treinados, a TDF é dependente do tipo e da velocidade de contração utilizados. Os maiores valores de TDF são obtidos quando não há movimento articular ou quando a velocidade de contração é baixa. Esses dados suportam a hipótese de que maiores velocidades de contração acarretam maior inibição do drive neural no início do movimento, diminuindo o torque e a TDF, principalmente nos primeiros 50ms de contração. As menores correlações entre a TDF isométrica e a concêntrica em alta velocidade $\left(180^{\circ} . \mathrm{s}^{-1}\right)$ sugerem que os fatores neuromusculares que as determinam podem ser diferentes.

Todos os autores declararam não haver qualquer potencial conflito de interesses referente a este artigo.

\section{REFERÊNCIAS BIBLIOGRÁFICAS}

1. Komi PV. Força e potência no esporte. 2ª ed. Artmed: Porto Alegre, 2006

2. Aagaard $P$, Simonsen EB, Andersen JL, Magnusson P, Dyhre-Poulsen P. Increased rate of force development and neural drive of human skeletal muscle following resistance training. J Appl Physiol. 2002;93:1318-26

3. Hakkinen K, Alen M, Komi PV. Changes in isometric force- and relaxation-time, electromyographic and muscle fibre characteristics of human skeletal muscle during strength training and detraining. Acta Physiol Scand. 1985;125:573-85.

4. Harridge SD, Bottinelli R, Canepari M, Pellegrino MA, Reggiani C, Esbjornsson M, et al. Whole-muscle and single-fibre contractile properties and myosin heavy chain isoforms in humans. Pflugers Arch. 432; $913-20$.

5. Thorstensson A, Grimby G, Karlsson J. Force-velocity relations and fiber composition in human knee extensor muscles. J Appl Physiol. 1976;40:12-6.

6. Aagaard P, Andersen JL. Correlation between contractile strength and myosin heavy chain isoform composition in human skeletal muscle. Med Sci Sports Exerc. 1998;30:1217-22.

7. Aagaard P. Training-induced changes in neural function. Exerc Sport Sci Rev. 2003;31:61-7.

8. Hakkinen K, Komi PV, Alen M. Effect of explosive type strength training on isometric force- and relaxation-time, electromyographic and muscle fibre characteristics of leg extensor muscles. Acta Physiol Scand. 1985;125:587-600.

9. Van Cutsem M, Duchateau J, Hainaut K. Changes in single motor unit behavior contributes to the increase in contraction speed after dynamic training in humans. J Physiol. 1998;513:295-305.

10. Thorstensson A, Karlsson J, Viitasalo HT, Luhtanen P, Komi PV. Effect of strength training on EMG of human skeletal muscle. Acta Physiol Scand. 1976;98:232-36.

11. Sale DG. Neural adaptation to resistance training. Med Sci Sports Exerc. 1988;20:S135-S45.
12. Jensen RL, Ebben WP. Quantifying plyometric intensity via rate of force development, knee joint, and ground reaction forces. J Strength Cond Res. 2007;21:763-7.

13. Bojsen-Møller J, Magnusson SP, Rasmussen LR, Kjaer M, Aagaard P. Muscle performance during maximal isometric and dynamic contractions is influenced by the stiffness of the tendinous structures. J Appl Physiol. 2005;99:986-94.

14. Wilson GJ, Lyttle AD, Ostrowski KJ, Murphy AJ. Assessing dynamic performance: a comparison of rate of force development tests. J Strength Cond Res. 1995;9:176-81.

15. Viitasalo JT, Komi PV. Force-time characteristics and fiber composition in human leg extensor muscles. Eur J Appl Physiol. 1978;40:7-15.

16. Baker D, Wilson G, Carlyon B. Generality versus specificity: a comparison of dynamic and isometric measures of strength and speed strength. Eur J Appl Physiol. 1994;68:350-5.

17. Westing SH, Seger JY. Eccentric and concentric torque-velocity characteristics, torque output comparisons, and gravity effect torque corrections for the quadriceps and hamstring muscles in females. Int J Sports Med. 1989;10:175-80.

18. Westing SH, Cresswell AG, Thorstensson A. Muscle activation during maximal voluntary eccentric and concentric knee extension. Eur J Appl Physiol Occup Physiol. 1991;62:104-8.

19. Dvir Z. Isocinética. Avaliações musculares, interpretações e aplicações clínicas. 1aㅡ. Ed. Manole: São Paulo, 2002.

20. Knapik J, Ramos M. Isometric and isokinetic torque relationships in the human body. Arch Phys Med Rehabil. 1980;61:64-7.

21. Folland JP, Willians AG. The adaptation to strength training: Morphological and neurological contributions to increased strength. Sports Med. 2007;37:145-68. 